Journal of Thermal Engineering, Vol. 5, No. 1, pp. 93-99, January, 2019

Yildiz Technical University Press, Istanbul, Turkey

\title{
PARTICIPATING MEDIA FOR VOLUMETRIC HEAT GENERATION
}

\author{
Layth Al-Gebory $1,2, *$
}

\section{ABSTRACT}

When an electromagnetic wave interacts with participating media (e.g. particulate media); the intensity of the radiation may be changed by the absorption, emission and scattering phenomena. Subsequently, the incident radiation will be attenuated and/or augmented under the effect of these phenomena. Light scattering and absorption by small particles are quite important in a wide range of applications such as meteorology, biomedicine, biophysics, astronomy, combustion, fire and flame, and solar thermal applications. Particulate media have been introduced as a working medium to improve the efficiency of thermal systems such as solar thermal power plants. The efficiency of the direct absorption solar thermal collectors (DASC) can be improved by using particulate media because of the unique thermo-optical properties, which in turn leads to enhance the thermal performance. The main objective of the present study is to investigate the effect of the participating media in the volumetric heat generation under the concept of photo-thermal energy conversion.

\section{Keywords: Participating Media, Volumetric Heat Generation}

\section{INTRODUCTION}

In the field of solar radiation utilization, solar thermal collectors are the most important parts of the solar thermal systems. However, the efficiency of these collectors is limited by the absorption properties of the working fluids, particularly water. Nanoparticle suspensions have been introduced as working media in solar thermal collectors to improve thermal efficiency and to reduce the size of such systems (energy efficient solar collectors) by using light induced energy conversion technique in a volume of suspended nanoparticles. It refers to direct absorption solar radiation (volumetric photo-thermal energy conversion), as shown in Figure 1. This figure shows the principles of photo-thermal energy conversion, where the incident solar radiation is directly absorbed by a volume of fluid which includes suspended particles. Then this energy is exploited in thermal applications. This method is one of the efficient methods in the field of energy conversion and production.

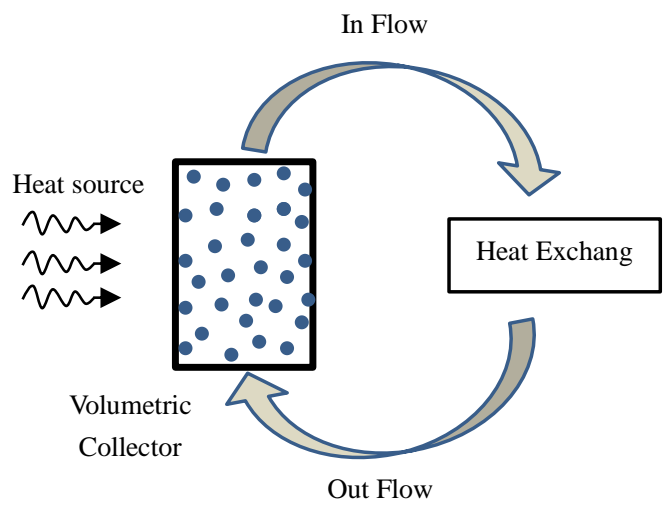

Figure1. Energy harvesting system

This paper was recommended for publication in revised form by Regional Editor Omid Mahian

${ }^{1}$ Department of Mechanical Engineering, Ozyegin University, Istanbul, Turkey

${ }^{2}$ Department of Materials Engineering, University of Technology, Baghdad, IRAQ

^E-mail address: layth.ismael@ozu.edu.tr, 130006@uotechnology.edu.iq

Orcid id: https://orcid.org/0000-0002-5943-7763

Manuscript Received 28 May 2017, Accepted 08 August 2017 
Nanoparticle suspensions are found to be effective electromagnetic wave absorbers within UV-Visible wavelength ranges where $85 \%$ of solar energy is dissolved. While conventional base fluids absorb $15 \%$ of solar energy within infrared wavelength range, nanoparticles have unique thermal and optical properties which are the basis of thermal applications [1-3]. In the field of particle suspension, long term stability of suspended nanoparticles is the key for any thermal application which includes such type of suspensions [4].

Owing to the advancement in nanotechnology during the past 20 years or so, applications of nanoparticles in solar thermal systems have been proposed. Compared to larger particles, the suspension with nanoparticles exhibits better stability with unique performance. In addition, the clogging and fouling would be less significant for suspension with nanoparticles [5, 6]. Absorption of light within the nanoparticle suspensions, largely by the nanoparticles, leads to a temperature rise in the suspensions that can then be exploited as thermal energy.

For that, the radiative properties of the suspended particles and the media that contains those particles should be investigated deeply. As there are many particles in a particulate medium, the scattered photon from a particle may interact with those from other particles. This means, an incident beam can be scattered more than one time and they can be scattered again by the nearby particles [7, 8].

The potential of using nanoparticles in the direct absorption solar collectors was first proposed by Tyagi et al. [9], they compared the collectors' performance using water/Al nanofluid as the working media. The radiative properties of water/ $/ \mathrm{TiO}_{2}$ nanofluid were investigated by Said et al. [10], the transmissivity of the nanofluid was achieved up to $60 \%$ in the particle volume fraction $0.08 \%$, and it was proven that the water/ $/ \mathrm{TiO}_{2}$ nanofluid could be a good option for the direct absorption solar collectors. More recently, the radiative properties are measured for the different metal oxide nanoparticles under the effects of temperature and particle concentration [11, 12]. Several nanofluids were prepared for this purpose, water based with metal oxide $\left(\mathrm{TiO}_{2}, \mathrm{Al}_{2} \mathrm{O}_{3}, \mathrm{ZnO}, \mathrm{CuO}\right.$, and $\left.\mathrm{Fe}_{2} \mathrm{O}_{3}\right)$ were investigated, and results conducted that the water $/ \mathrm{TiO}_{2}$ nanofluid with $0.05 \%$ volume fraction required minimum pumping power and show good radiative properties.

Through the analysis of the optical and thermal behavior of particulate media, it is clear that photo-thermal energy conversion is important to not only the solar thermal systems but also to the electric power generation and solar chemical technology. This research investigates the effects of nanoparticle suspensions (water/ $\mathrm{TiO}_{2}$ nanoparticle suspension) at different particle concentrations on the radiative properties and radiative transfer phenomena. The effect and contribution of the $\mathrm{TiO}_{2}$ nanoparticles on the radiative properties in the UV-Vis-NIR wavelength ranges are observed, which have a significant impact for the solar thermal applications.

\section{THEORY}

The principle of the photo-thermal energy conversion is converting the energy of the incident radiation to thermal energy, solar thermal systems collectors are one of the examples. The energy equation for the solar system collector, accounting for the volumetric heat release, can be written as:

$$
\rho_{f} C_{p_{f}} u \frac{\partial T}{\partial x}=k_{f} \frac{\partial^{2} T}{\partial y^{2}}+S_{e}+q_{r a d}^{\prime \prime \prime}
$$

where $\rho_{f}, C_{p_{f}}, k_{f}, T, u$ and $q_{r a d}^{\prime \prime \prime}$ are the density, specific heat, thermal conductivity, temperature, the velocity of the medium, and volumetric heat generation respectively. The source term $\left(S_{e}\right)$ in Equation 1 represents the energy transfer between the medium and the suspended particles (between the phases) [13,14]:

$$
S_{e}=\sum_{n p} \frac{m_{p}}{\delta V} C_{p_{p}} \frac{d T_{p}}{d t}
$$

where the subscript $(p)$ refers to the nanoparticles and $(V)$ is the volume occupied the nanosuspension. 
In order to determine the volumetric heat generation inside a participating media, the spectral intensity must be integrated over the entire spectrum within all wavelengths and directions $[15,16]$.

$$
q_{\text {rad }}^{\prime \prime \prime}=\int_{0}^{\pi / 2} \int_{0}^{\infty} \int_{0}^{2 \pi} I_{\lambda}(\hat{\boldsymbol{s}}) \cos \theta \sin \theta d \theta d \lambda d \phi
$$

It is usually to describe the direction vector in terms of a spherical or polar coordinate system. Consider a point $P$ on an opaque surface $d A$ radiating into another medium, as shown in Figure 2. This figure shows the radiation propagation in a hemisphere to demonstrate the incident field.

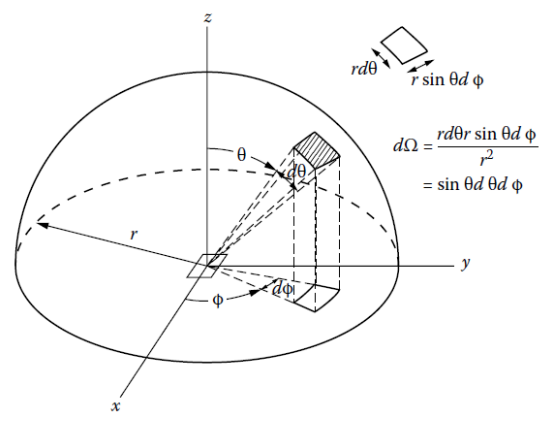

Figure 2. Hemisphere shows the direction of incident radiation and solid angle relations [16].

The radiative transfer equation (RTE) in its quasi-steady form requires the knowledge of the radiative intensity $\left(I_{\lambda}\right)$ at a single point in space into the direction of $(\widehat{\mathrm{s}})$, can be written as $[15,16]$ :

$$
\frac{\mathrm{dI} \lambda}{\mathrm{ds}}=-\beta_{\lambda} \mathrm{I}_{\lambda}(\hat{\mathrm{s}})+\kappa_{\lambda} \mathrm{I}_{\mathrm{b} \lambda}+\frac{\sigma_{\text {sca. } \lambda}}{4 \pi} \int_{4 \pi} \mathrm{I}_{\lambda}(\hat{\mathrm{s}}) \Phi_{\lambda}\left(\hat{\mathrm{s}}_{\mathrm{i}}, \hat{\mathrm{s}}\right) \mathrm{d} \Omega
$$

In diluted suspensions (low particles concentration) the scattered intensity of a particulate suspension is equal to the scattered intensity from a single particle multiple by the number of the particles, which is known as the linear summation rule. This rule is applicable and valid for most radiative transfer applications, where scattering and absorption are treated independently [15,17].

The size parameter $(x)$, the relative complex refractive index of the nanosuspension $(m)$, can be estimated using the following relation:

$$
m=\frac{n_{\text {particle }}}{n_{\text {medium }}}
$$

The scattering and absorption coefficients of the suspended nanoparticles can be calculated as:

$$
\begin{gathered}
\sigma_{\text {scat. }}=\frac{3}{2} \frac{\emptyset\left(Q_{\text {scat }}\right)}{d_{p}} \\
\sigma_{\text {abs. }}=\frac{3}{2} \frac{\emptyset\left(Q_{a b s}\right)}{d_{p}}
\end{gathered}
$$

The extinction coefficient (scattering and absorption) of the nanoparticles can be calculated as: 


$$
\sigma_{e x t, p}=\sigma_{s c a t, p}+\sigma_{a b s, p}
$$

The medium absorption should be taken into the consideration. Therefore, any absorption of the medium must be incorporated in the calculations. Then, the extinction coefficient is obtained from [18]:

$$
\sigma_{\text {ext }, m}=\frac{4 \pi k_{\text {medium }}}{\lambda}
$$

where $k_{\text {medium }}$ is the absorption index of the medium which is wavelength dependent.

The total extinction coefficient of a nanosuspension when combined is as given below:

$$
\sigma_{\text {total }}=\sigma_{e x t, p}+\sigma_{e x t, m}
$$

\section{RESULTS AND DISCUSSION}

Figure 3 shows a transparent enclosure includes a particulate (participating) media. This figure describes the radiative phenomena inside a particulate media. When radiative energy travels through participating media, the incident beams are attenuated by scattering and absorption, while others are transmitted through this media to the other side.

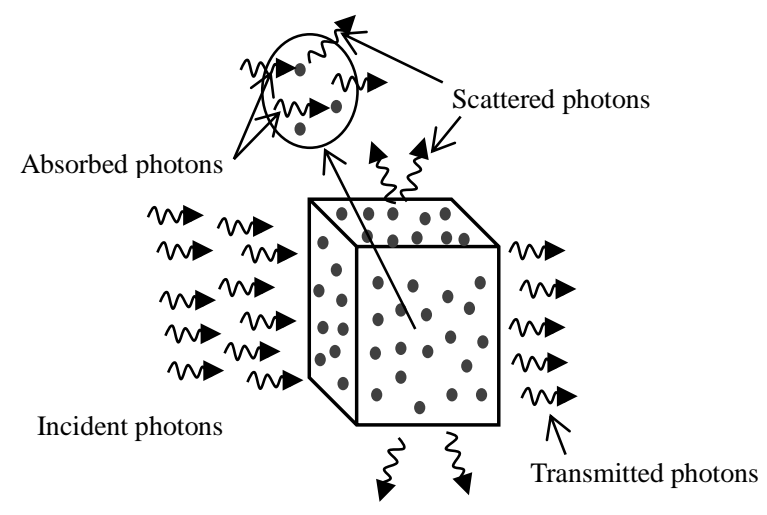

Figure 3. Incidence of radiative energy on a particulate media

In the present research, the results are obtained for water based $\mathrm{TiO}_{2}$ nanosuspensions. The effects of suspended nanoparticle size $\left(d_{p}=50 \mathrm{~nm}\right)$ in different particle volume fractions on the radiative properties are theoretically investigated.

Figure 4 shows the scattering coefficient of the water based $\mathrm{TiO}_{2}$ nanosuspensions. The effect of particles volume fraction $(0.01,0.06$ and $0.1 \%)$ on the scattering behavior is explained. It can observe that particles with small size have scattering coefficient approaches to zero in the longer wavelength ranges; almost when the wavelength approaches or longer than $600 \mathrm{~nm}$. The effect of the particles is shown clearly at short wavelength ranges. As the number of suspended particles (particle concentration) increase, the scattering coefficient increases. The relationship between the particle size and the incident wavelength is one of the most important parameters which affect the radiation transfer phenomena. Therefore, nanoparticles play an important role in the UV-Vis wavelength ranges.

The extinction coefficient (scattering and absorption) of the nanosuspensions is shown in Figure 5. In this figure, the scattering effect comes from the effect of the particles where the medium (water) contributes by the absorption effect. The absorption coefficient is included in the infrared wavelength range, where water has low absorption index in the UV-Vis wavelength ranges. Particle scattering in the UV-Vis wavelength ranges can enhance 
the extinction coefficient of the media. Indeed, the radiative properties of the particles and the medium are the basis of the direct absorption solar collectors, where the energy of the incident radiation (electromagnetic energy) is converted to the thermal energy. The extinction coefficient refers to the absorption of the incident radiation by the participating media.

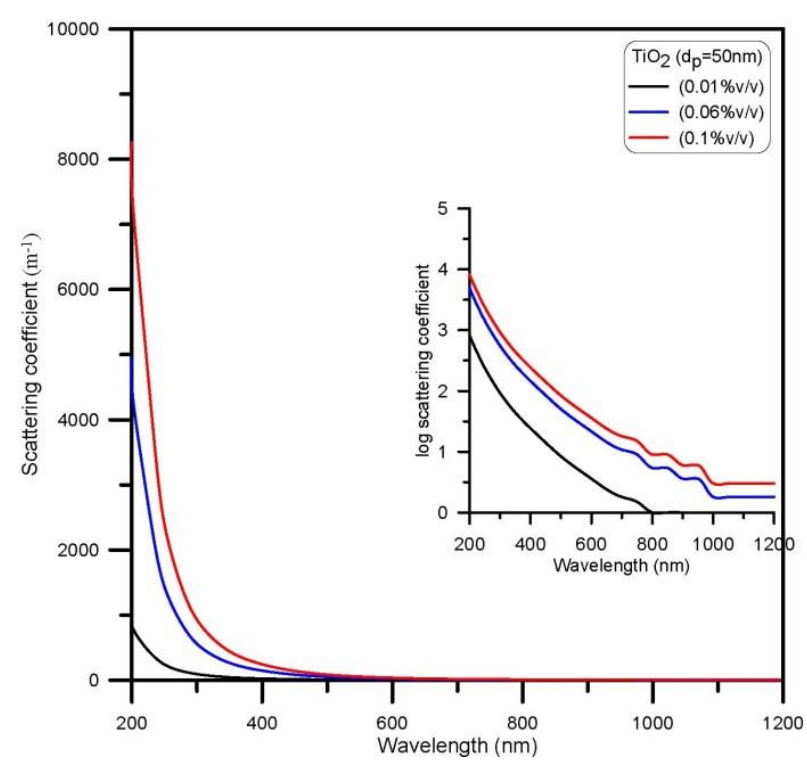

Figure 4. Scattering coefficient of water based $\mathrm{TiO}_{2}\left(d_{p}=50 \mathrm{~nm}\right)$ nanosuspensions

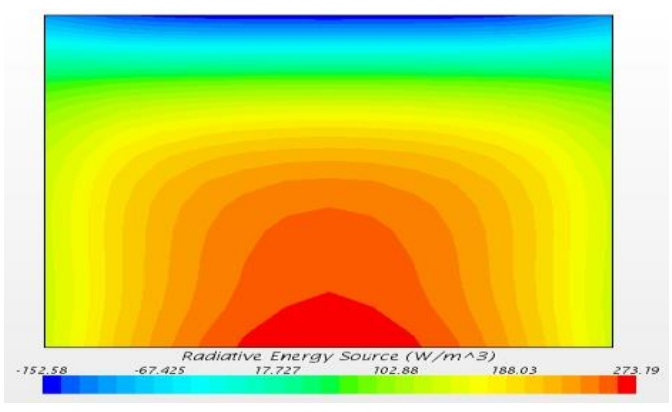

(a)

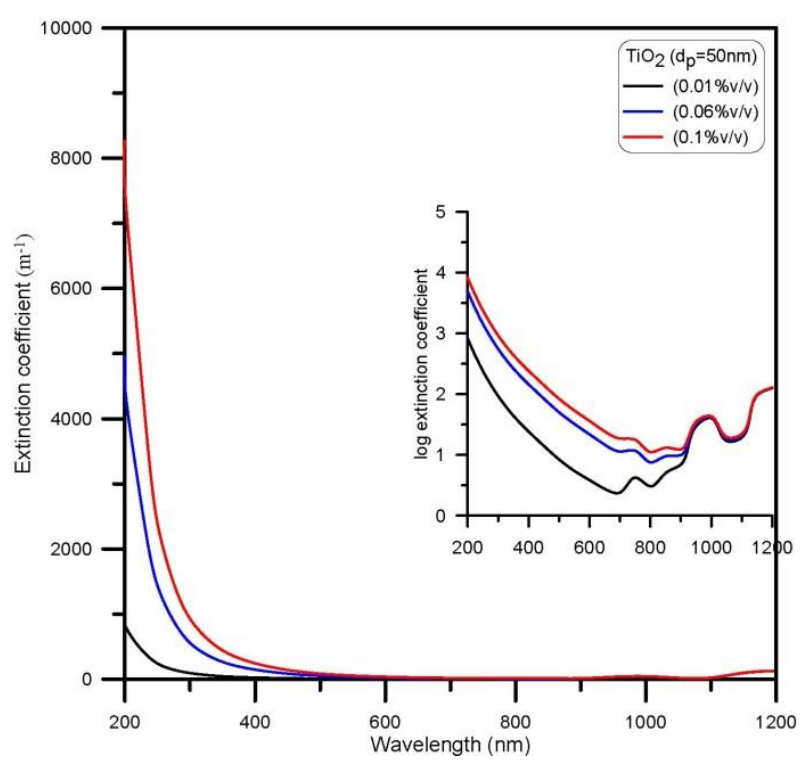

Figure 5. Extinction coefficient of water based $\mathrm{TiO}_{2}\left(d_{p}=50 \mathrm{~nm}\right)$ nanosuspensions

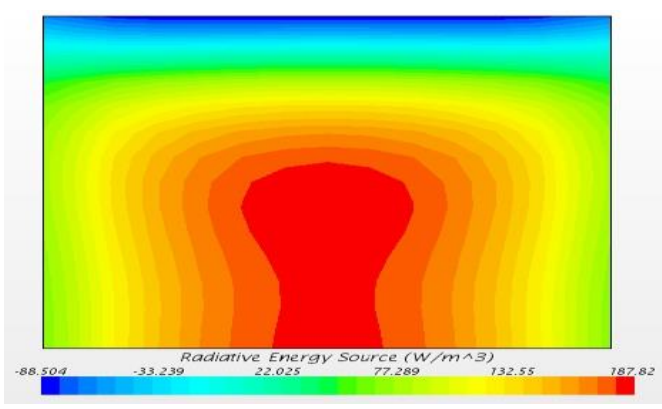

(b)

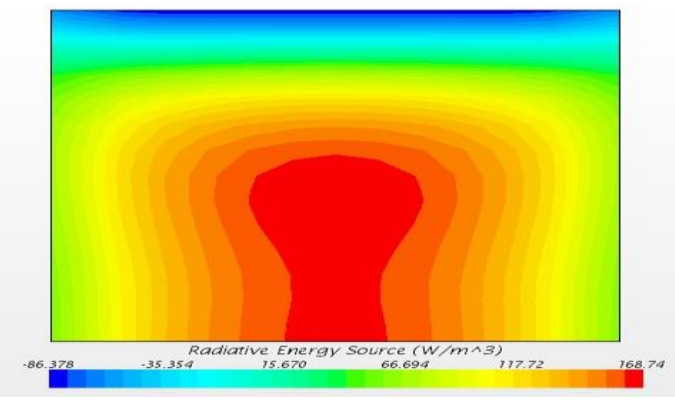

(c)

Figure 6. Radiative energy source in $\mathrm{TiO}_{2}\left(d_{p}=50 \mathrm{~nm}\right.$ ) nanosuspension. (a) $0.01 \% \mathrm{v} / \mathrm{v}$, (b) $0.06 \% \mathrm{v} / \mathrm{v}$ and (c) $0.1 \%$ v/v 
The incident radiation on a participating media undergoes two phenomena: attenuation and augmentation. Radiation attenuation comes from the effects of absorption and out-scattering (the deviation of the incident radiation to a direction other than the incident direction). On the other hand, radiation augmentation includes the effects of the re-radiation (emission) and in-scattering (increasing the scattering in the direction of the incident radiation). These two phenomena (attenuation and augmentation) are explained by the radiative energy source, which indicates the absorption behavior of a participating media.

Figure 6 shows the radiative energy source which is generated in the volume of participating media (nanoparticulate media). The boundaries of the volume are specified as follows: the upper face is a radiant source $\left(1400 \mathrm{~W} / \mathrm{m}^{2}\right)$, the lower face is adiabatic and reflects the incident radiation inside the volume of the nanosuspension, the side surface is adiabatic surfaces. The main objective is to observe the effect of the nanoparticles on the radiative transfer phenomena and to clarify the effect of particle concentration on the radiative heat generation in the participating media. The generated radiative energy inside a volume of participating media is one of the thermal applications which are desired for different engineering and industrial applications. The spectral properties of the participating media play a significant role in these applications.

\section{CONCLUSION}

Suspended nanoparticles play a significant role in a wide range of applications because of their thermal and optical properties. Particle size and size distribution show significant effects in different wavelength ranges, which is very important in specific applications including solar thermal systems in addition to other applications. Radiative properties (scattering and absorption) carry the significant impact on the thermal radiative transfer, where the energy of the incident radiation is converted directly to thermal radiation. Different nanoparticles in different conditions show different radiative properties, which can be exploited in the thermal field for specific applications.

The aim of the using participating media is not only to convert concentrated radiation energy into thermal energy as efficiently as possible but also, to achieve the highest possible temperatures while retaining this high thermal efficiency.

In this study, it can observe that the nanoparticles play a significant role in the UV-Vis wavelength ranges where a large amount of the solar energy is dissipated. The extinction coefficient of the media is enhanced and affects the radiation transfer performance. Particle size and number have important effects on the thermal radiation transfer, increasing particle concentration leads to enhance the radiative properties.

\section{NOMENCLATURE}

$\begin{array}{ll}C_{p_{f}} & \text { Specific heat, } \mathrm{kJ} / \mathrm{kg}{ }^{\circ \mathrm{C}} \\ d_{p} & \text { Particle diameter, } \mathrm{m} \\ I_{\lambda} & \text { Spectral radiation intensity, } \mathrm{W} /\left(\mathrm{m}^{2} \mu \mathrm{m} \mathrm{sr}\right) \\ k & \text { Absorption inex, nd } \\ k_{f} & \text { Thermal conductivity, } \mathrm{W} / \mathrm{m}{ }^{\circ} \mathrm{C} \\ m & \text { Relative complex refractive index, } \mathrm{nd} . \\ n & \text { Refractive index, nd } \\ q_{r a d}^{\prime \prime \prime} & \text { Volumetric heat generation, } \mathrm{W} / \mathrm{m}^{3} \\ Q & \text { Radiative efficiency factor, nd } \\ t & \text { Time, sec } \\ \widehat{\mathrm{s}} & \text { Unit vector in the } \Omega \text { direction, } \mathrm{nd} \\ S_{e} & \text { Source term } \\ T & \text { Temperature, }{ }^{\circ} \mathrm{C} \\ u & \text { Velocity of the medium, m/sec. } \\ V & \text { Volume occupied the nanosuspension, } \mathrm{m}^{3} \\ x & \text { Size parameter, nd }\end{array}$

Greek symbols 


$\begin{array}{ll}\beta & \text { Extinction or attenuation coefficient, } \mathrm{m}^{-1} \\ \theta & \text { Polar or cone angle measured from normal of surface, rad. } \\ \rho_{f} & \text { Density of a fluid. } \mathrm{kg} / \mathrm{m}^{3} \\ \sigma_{\text {scat }} & \text { Scattering coefficient } \mathrm{m}^{-1} \\ \sigma_{\text {abs }} & \text { Scattering coefficient, } \mathrm{m}^{-1} \\ \phi & \text { Azimuthal angle, rad. } \\ \Phi & \text { Scattering phase function } \\ \Omega & \text { Solid angle, sr } \\ & \\ \text { Subscripts } & \\ f & \text { Refers to fluid } \\ m & \text { Refers to medium } \\ p & \text { Refers to nanoparticle }\end{array}$

\section{REFERENCES}

[1] Duffie J. A. and Beckman W. A. (1991). Solar Engineering of Thermal Processes, $2^{\text {nd }}$ edition, John Wiley \& Sons, New York.

[2] Green M. A. (1981). Solar Cells: Operation Principles, Technology, and System Applications, Prentice Hall, Englewood Cliffs, NJ.

[3] Choi C., Yoo H. S. and Oh J. M. (2008). Preparation and heat transfer properties of nanoparticle-in-transformer oil dispersions as advanced energy-efficient coolants, Current Applied Physics 8 710-712.

[4] Luo, Z., Wang, C., Wei, W., Xiao, G., \& Ni, M. (2014). Performance improvement of a nanofluid solar collector based on direct absorption collection (DAC) concepts. International Journal of Heat and Mass Transfer, 75, 262271.

[5] Myers, D. (1999). Wetting and spreading. In Surfaces, interfaces and colloids. Principles and applications (pp. 415-447). John Wiley \& Sons, New York.

[6] Kozan, M., Thangala, J., Bogale, R., Mengüç, M. P., \& Sunkara, M. K. (2008). In-situ characterization of dispersion stability of $\mathrm{WO}_{3}$ nanoparticles and nanowires. Journal of Nanoparticle Research, 10(4), 599-612.

[7] Mischenko, M. I., Wiscombe, W. J., Hovenier, J. W., \& Travis, L. D. (2000). Overview of scattering by nonsperical particles.

[8] Du, M., \& Tang, G. H. (2015). Optical property of nanofluids with particle agglomeration. Solar Energy, 122, 864-872.

[9] Tyagi, H., Phelan, P., \& Prasher, R. (2009). Predicted efficiency of a low-temperature nanofluid-based direct absorption solar collector. Journal of solar energy engineering, 131(4), 041004.

[10] Said, Z., Sajid, M. H., Saidur, R., Mahdiraji, G. A., \& Rahim, N. A. (2015). Evaluating the optical properties of $\mathrm{TiO}_{2}$ nanofluid for a direct absorption solar collector. Numerical Heat Transfer, Part A: Applications, 67(9), 10101027.

[11] Milanese, M., Colangelo, G., Cretì, A., Lomascolo, M., Iacobazzi, F., \& De Risi, A. (2016). Optical absorption measurements of oxide nanoparticles for application as nanofluid in direct absorption solar power systems-Part I: water-based nanofluids behavior. Solar Energy Materials and Solar Cells, 147, 315-320.

[12] Milanese, M., Colangelo, G., Cretì, A., Lomascolo, M., Iacobazzi, F., \& De Risi, A. (2016). Optical absorption measurements of oxide nanoparticles for application as nanofluid in direct absorption solar power systems-Part II: $\mathrm{ZnO}, \mathrm{CeO} 2, \mathrm{Fe}_{2} \mathrm{O}_{3}$ nanoparticles behavior. Solar Energy Materials and Solar Cells, 147, 321-326.

[13] Goutam, S., \& Paul, M. C. (2014). Discrete phase approach for nanofluids flow in pipe.

[14] Diggs, A., \& Balachandar, S. (2016). Evaluation of methods for calculating volume fraction in EulerianLagrangian multiphase flow simulations. Journal of Computational Physics, 313, 775-798.

[15] Modest M. F., "Radiative Heat Transfer", Academic Press-Elsevier science”, USA, 2003.

[16] Howell, J. R., Menguc, M. P., \& Siegel, R. (2015). Thermal radiation heat transfer. CRC press.

[17] Fan, L. S., \& Zhu, C. (1998). Principles of Gas-Solid Flows, Cambridge Series in Chemical Engineering. Cambridge University Press, United Kingdom.

[18] Otanicar, T., Taylor, R. A., Phelan, P. E., \& Prasher, R. (2009, January). Impact of size and scattering mode on the optimal solar absorbing nanofluid. In ASME 2009 3rd International Conference on Energy Sustainability collocated with the Heat Transfer and InterPACK09 Conferences. American Society of Mechanical Engineers, 791-796. 